SHS Web of Conferences 10, 00025 (2014)

DOI: $10.1051 /$ shsconf $/ 20141000025$

C Owned by the authors, published by EDP Sciences, 2014

\title{
Physical activity in physiotherapy and physical education high school students
}

\author{
A. Mihailova, I. Kaminska, and A. Bernane \\ Daugavpils University, Latvia
}

\begin{abstract}
A term of health-related physical fitness became topical with four its components: aerobic and/or cardiovascular fitness, body composition, abdominal muscle strength and endurance, and lower back and hamstring flexibility. Complex evaluation of health-related physical fitness and physical activity (PA) may show a wider insight in health promotion and disease prevention. The aim of this study was to evaluate physical activity relation to health-related physical fitness in Physiotherapy (PT) and Physical Education (PE) students. Final study sample consisted of 67 students (46 women and 21 men) (aged $21.61 \pm 0.71$ ). All participants filled in International Physical Activity Questionnaire. Health-related physical testing included: 1) body composition evaluation, 2) abdominal muscles strength tests, 3) dynamometry, 4) hamstring muscles and m. quadratus lumborum elasticity evaluation tests, 5) bicycle ergometer test (anaerobic threshold, maximal oxygen consumption). Results showed that most students had normal body composition parameters (BMI, body fat, muscle mass, body water) in both genders and study programs. Women were less physically active that men, and PA duration was higher in PE students. PT students had higher body composition values, lower cardiorespiratory fitness parameters and lower handgrip strength in both hands than PE students. Greater PA generally implies a higher level of health-related physical fitness. PA significantly positively affects body composition, upper $m$. rectus abdominis strength, grip strength and aerobic capacity.
\end{abstract}

\section{Introduction}

Cross-sectional and longitudinal research has established a positive relationship between participation in moderate-to-vigorous physical activity (PA) and increase in cardiovascular and musculoskeletal fitness, weight management, and reduction of adult-like risk factors such as obesity and high blood pressure for chronic diseases (Beets and Piletti, 2005; Ruiz et al., 2006; Mesa et al., 2006; Moliner-Urdiales et al., 2010; Cheng and Yang, 2011). Several studies have demonstrated an association between the level of physical fitness during childhood and adolescence and cardiovascular risk factors (Ortega et al., 2005; Wedderkopp et al., 2003; Ekelund et al., 2001; Nielsen and Andersen, 2003). Likewise, important longitudinal studies have shown that the level of physical fitness in an adult, as well as the presence of other conventional cardiovascular risk factors, such as hypercholesterolemia or hypertension, is conditioned by the level of physical fitness in childhood or adolescence (Ortega et al., 2005; Janz, Dawson and Mahoney, 2002; Boreham et al., 2001; 2002; Haseelstrom et al., 2002; Twisk, Kemper and

This is an Open Access article distributed under the terms of the Creative Commons Attribution License 4.0, which permits unrestricted use, distribution, and reproduction in any medium, provided the original work is properly cited. 
Van Mechlen, 2002). Thus, it's important to start evaluating PA and physical fitness in youth, in order to manage health promotion and make preliminary evaluation of health-related parameters.

The Centers for Disease Control and Prevention developed standard definition for the term physical fitness in 1985: “... a set of attributes or characteristics that people have or achieve that relates to the ability to perform physical activity" (ACSM, 2010). Health-related physical fitness (HRPF) is a more specific term defined by President's Council on Physical Fitness as consisting of those specific components of physical fitness that have relationship with good health (President's Council on Physical Fitness, USA) (ACSM, 2010). HRPF refers to cardiorespiratory fitness, muscular strength, flexibility and body composition (Cheng et al., 2011; ACSM, 2010; Moliner-Urdiales et al., 2010). Among the important reasons for assessing of HRPF are: 1) educating participants about their HRPF status; 2) providing data that are helpful in the development of exercise prescriptions to address all fitness components; 3) collecting baseline and follow-up data that allow evaluation of progress by exercise program participants; 4) motivating participants by establishing reasonable and attainable fitness goals; 5) stratifying cardiovascular risk (ACSM, 2010).

University time is considered a critical period in an individual's lifetime. The leisure activities in which university students participate, the lifestyle habits they have, and the exercise habits they form all have significant impacts on their health, university life and on their work life after graduation (Cheng et al., 2011). University life usually only gives an opportunity to attend several sport classes (aerobics, swimming etc.) using university's sport facilities, but as research data show not all students uses this opportunity or have additional sport activities apart studies. Research data of Latvian researchers with J. Porozovs (2012) in lead has showed that only $15 \%$ of students have do regular sport exercises comparing with $42 \%$ or students who didn't do any sport at all.

Typically PA decreases with age, but not among those who exercise regularly. Although regular exercise decreases after the 15-24 age groups (32\%), it then stabilizes at 26-27\% for all other age categories. This clearly shows that the majority of those who form a regular exercise pattern early on carry it on throughout their lives. The data suggests the importance of setting a pattern of exercising early on in life, as the majority of people then appear to continue exercising well into their retirement. Only $7 \%$ of $15-24$ year-olds never exercise at all, as opposed to $27 \%$ of respondents in the $70+$ group (Kundziòa, Grants, 2011).

In this study we examined PA and HRPF of university students. The aim of the study was to evaluate PA relation to HRPF in Physiotherapy and Physical Education students.

\section{Materials and methods}

A total of 91 students of Daugavpils University were recruited. During the analysis phase, 24 participants were excluded because they didn't fit any of inclusion criteria (adolescents aged 19 to 25 years old, students of Physiotherapy (PT) and Physical Education (PE) and absence of contradictions to any of HRPF tests) or had missed health-related physical fitness tests or hadn't filled in the questionnaire. The final study group consisted of 67 students (46 women and 21 men) with an age range of 20 to 23 years (mean age $21.61 \pm 0.71$ years).

Physical activity was assessed with International Physical Activity Questionnaire (IPAQ) - Long Form (www.ipaq.ki.se). Three levels of physical activity were established according to IPAQ Scoring Protocol guidelines: low, moderate and high (Patterson, 2010).

Body composition was assessed by the bioelectrical impedance analysis method using medically approved portable body composition monitor with visceral fat indicator TANITA BC-420. Body weight, body mass index, body fat (\%), muscle mass $(\%)$ and body water $(\%)$ variables were received. Body height that was required to fill in the TANITA measuring protocol was measured to the nearest $1.0 \mathrm{~cm}$. Waist and hip girth were measured to the nearest $0.5 \mathrm{~cm}$ with a flexible leather tape measure. Waist girth was measured midway between the lowest rib and the iliac crest when subject were in standing position. 
Int. Conf. SOCIETY. HEALTH. WELFARE.

Table 1. M. quadratus lumborum elasticity by physical activity levels (PAL).

\begin{tabular}{|l|l|l|l|l|}
\hline \multirow{2}{*}{ M. quadratus lumborum } & \multicolumn{3}{|c|}{ Physical activity (PA) level } & \multirow{2}{*}{ Total } \\
\cline { 2 - 4 } & Low PA & Moderate PA & High PA & \\
\hline Symmetry & $3(4.48 \%)$ & $25(37.31 \%)$ & $22(32.84 \%)$ & $50(74.63 \%)$ \\
\hline Right side shortened & $2(2.99 \%)$ & $1(1.49 \%)$ & $4(5.97 \%)$ & $7(10.45 \%)$ \\
\hline Left side shortened & $1(1.49 \%)$ & $5(7.46 \%)$ & $4(5.97 \%)$ & $10(14.92 \%)$ \\
\hline Total & $6(8.96 \%)$ & $31(46.26 \%)$ & $30(44.78)$ & $67(100 \%)$ \\
\hline
\end{tabular}

Hip girth was measured at the highest points of gluteal muscles when subject were in standing position (Adams and Beam, 2008).

The grip strength of both hand was measured with hydraulic hand dynamometer (Saehan Corp., Korea, Model SH5001, SN 11010449) while the subjects stood with their elbows extended (Adams and Beam, 2008). The best score of 3 trials was recorded for analysis.

Abdominal muscles and hamstring muscles manual tests were performed on the basis of guidelines on muscle testing of Palmer M. Lynn and Marcia E. Epler "Fundamentals of Musculoskeletal Assessment Techniques" (1998). M. rectus abdominis upper and lower parts, $m$. obliquus abdominis externus et internus were tested for strength (evaluated from 0 to 5 points) and $m$. quadratus lumborum and hamstring muscles were tested for elasticity in terms of flexibility.

Cardiorespiratory fitness was assessed using cardiopulmonary testing system MasterScreenCPX (JAEGER) and cycle ergometer Ergoselect 100P (Ergoline, SN 2008000567) with breath-by-breath analysis of expired air. Subjects performed submaximal test based on the method of PWC170 testing with two power stages each of 5 minutes (Adams and Beam, 2008). Start power for both women and men was equivalent to $1 \mathrm{~W}$ per 1 kilogram of body weight for subject with normal BMI and $0.75 \mathrm{~W}$ per 1 kilogram of body weight for overweight subjects. Then power was increased according to the heart rate at the end of the first load stage. Maximal oxygen consumption and anaerobic threshold was calculated in MasterScreenCPX program automatically. They were taken as basic variables for evaluation of aerobic capacity.

Statistical analyses were performed using IBM SPSS Statistics (version 20.0). Group differences were assessed with Chi-Square, T-test and ANOVA, drawn at significant level of $0.05 \%$.

\section{Results}

Most students had normal body composition parameters (BMI, body fat, muscle mass, body water) in both genders and study programs. $69.6 \%$ of women and $71.4 \%$ of men met the criteria for normal BMI. The difference between BMI groups in women and men was not significant $(p=0.631) .71 .7 \%$ of women and $85.7 \%$ of men had normal body fat content. Distribution in BF groups did not differ among women and men $(p=0.439) .73 .9 \%$ of women and $85.7 \%$ of men and $93.5 \%$ of women and $100 \%$ of men met the criteria of good muscle mass and body water accordingly ( $p<0.05$ between genders).

$74.63 \%$ of students had $m$. quadratus lumborum symmetry, $37.31 \%$ of them was in moderate PAL group (Table 1). M. quadratus lumborum elasticity did not differ significantly between PAL groups $(p=0.237)$.

$46.26 \%$ of students had moderate PA level, $44.78 \%$ of students - high PA level (Table 1). Women were less physically active than men $(p<0.001)$ having a prevalence of moderate PAL $(63.0 \%)$ comparing with $90.5 \%$ of men in high PAL (Fig. 1). PE students were more physically active than PT students $(p=0.002)$. PE students had prevalence of high PAL $(95 \%)$ comparing with $66 \%$ of moderate PAL in PT students $(p<0.0001)$. Women of PE study program had a prevalence of high PAL, comparing with prevalence of moderate PA in women of PT study program $(p=0.004)$. Men of 


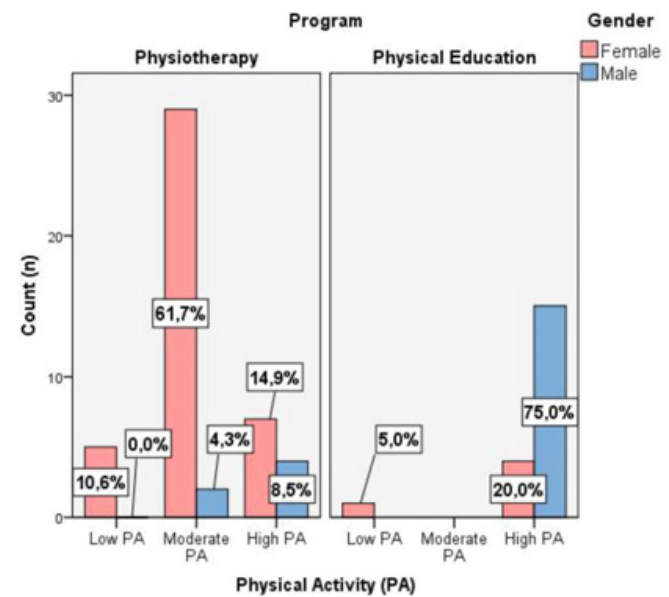

Figure 1. Physical activity levels by gender and study program.

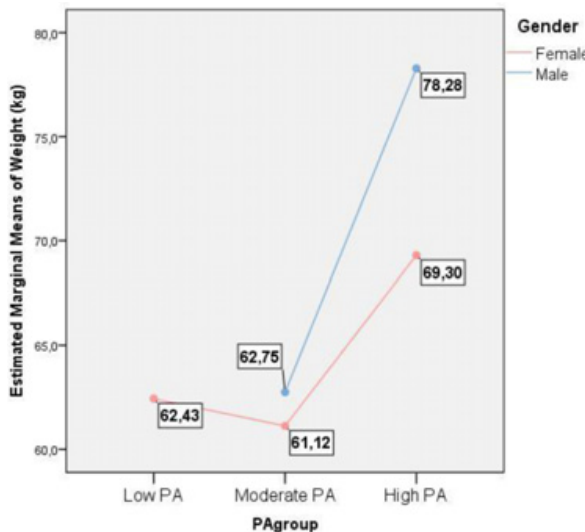

Figure 2. Mean values of body weight in different PAL groups in both genders.

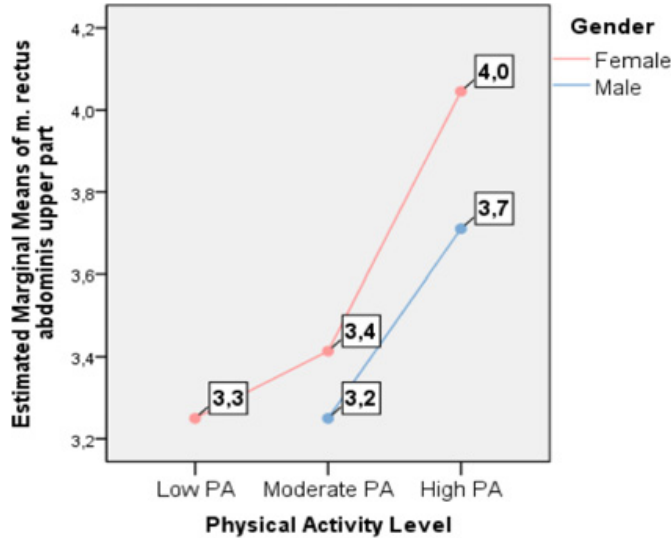

Figure 3. $M$. rectus abdominis upper part strength in PAL groups and genders.

PE study program were more physically active than men of PT study program, having a prevalence of high PAL $(p=0.019)$.

PE students had higher BMI ( $p=0.033)$, WHR $(p=0.0001)$, body water $(p=0.007)$ and muscle mass $(p=0.001)$ values, but lower body fat $(p=0.001)$. PT students had lower cardiorespiratory fitness parameters: anaerobic threshold $(p=0.0001)$, absolute $(p=0.0001)$ and relative $(p=0.001)$ maximal oxygen consumption and lower handgrip strength in both hands $(p<0.0001)$ than PE students. Musculoskeletal fitness values did not differ significantly between study programs.

UNIVARIATE analysis showed a significant relationship between weight and PAL. Students with high PAL had higher body weight comparing with those, who were insufficiently $(p=0.026)$ or moderate $(p<0.0001)$ physically active (Fig. 2).

$M$. rectus abdominis upper part strength (Fig. 3) was significantly higher in high PAL comparing with moderate PAL ( $p=0.027)$ in both genders. There's no statistical significant influence of PAL on obliques muscles strength in rotation to the right and left in both genders. 
Int. Conf. SOCIETY. HEALTH. WELFARE.

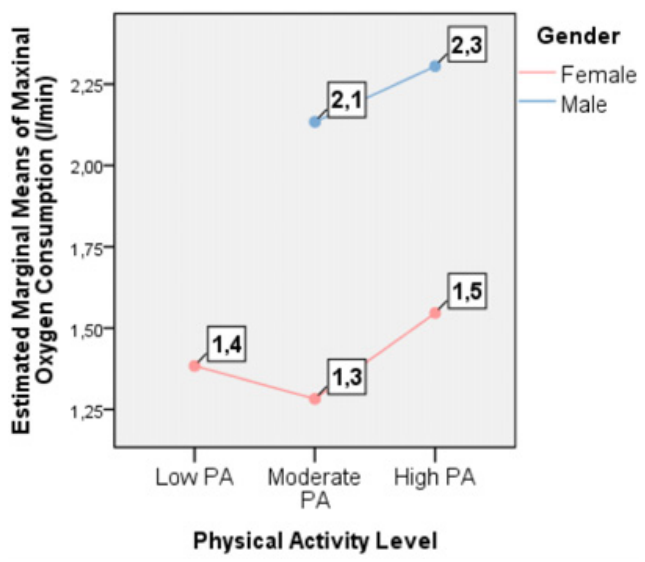

Figure 4. Absolute maximal oxygen consumption mean values in different PAL.
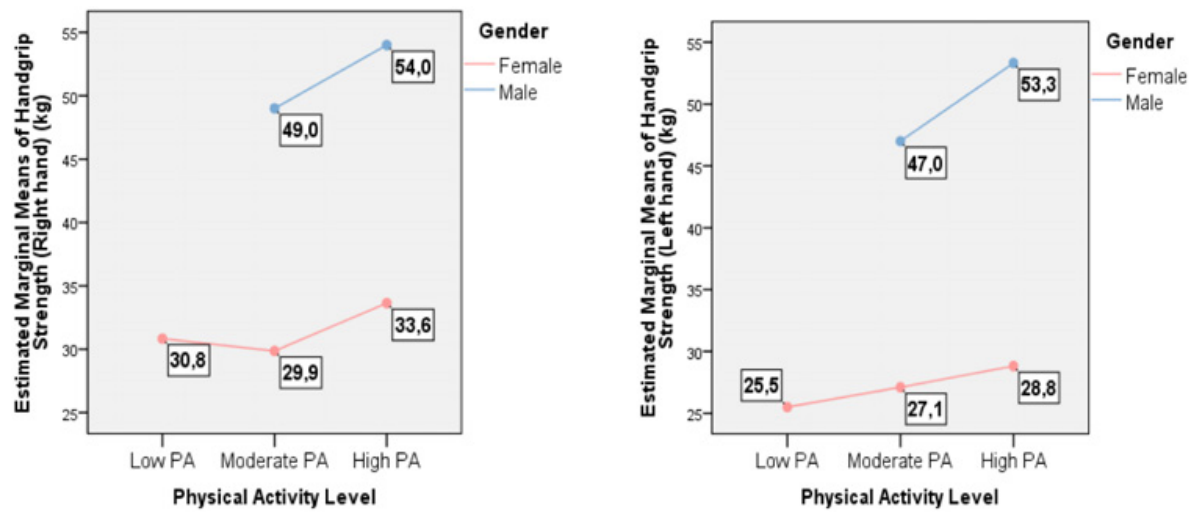

Figure 5. Handgrip strength values (right and left hands) in different PAL.

PAL had a significant effect on maximal oxygen consumption in both women and men. Absolute maximal oxygen consumption values were significantly higher in high PAL than in moderate PAL $(p=0.045)$ in women (Fig. 4). And gender was an independent factor affecting anaerobic threshold and relative maximal consumption values in all PAL $(p<0.0001)$.

Handgrip strength of both hands was significantly higher in high PAL group comparing with moderate PAL (right hand $\mathrm{p}<0.0001$, left hand $\mathrm{p}<0.0001$ ) in both genders (Fig. 5). Statistically significant difference was found for handgrip strength of left hand between low and high PAL $(\mathrm{p}<0.0001)$ in women (Fig. 5).

Physical activity weekly amount positively correlates with $m$. rectus abdominis upper part strength ( $r=0.330, p=0.025$ ), grip strength (right hand: $r=0.341, p=0.020$ ) in women when adjusted by gender. Vigorous intensity physical activity weekly amount significantly correlates with $m$. rectus abdominis upper part strength $(r=0.416, p=0.004)$, oblique muscles strength in rotation to the right and left, and handgrip strength of right hand in women. When not adjusted by age PA total weekly amount significantly correlates with cardiorespiratory fitness parameters: absolute maximal oxygen consumption $(r=0.310, p=0.011)$, relative maximal oxygen consumption $(r=242, p=0.048)$ and anaerobic threshold $(r=279, p=0.022)$. 
SHS Web of Conferences

\section{Discussion}

The purpose of this study was to evaluate physical activity relation to health-related physical fitness in Physiotherapy and Physical Education students. The result showed that greater physical activity was associated with a higher level of health-related physical fitness in students of Physiotherapy and Physical Education study programs. Specifically, the $m$. rectus abdominis upper part and oblique muscles strength in rotation to the right and left, handgrip strength and maximal oxygen consumption were higher in high PAL group women. Physical activity weekly amount was correlated positively with $\mathrm{m}$. rectus abdominis upper part strength, grip strength in women. When not adjusted by age total PA weekly amount significantly correlated with cardiorespiratory fitness parameters: absolute and relative maximal oxygen consumption, and anaerobic threshold.

Additionally, our findings showed that women were less physically active than men having a prevalence of moderate PAL comparing with $90.5 \%$ of high PAL in men. Our study findings are consistent with Canadian Fitness \& Lifestyle Research Institute (2002) data, that women are less physically active comparing with men. Only $36 \%$ of women comparing with $52 \%$ of men are physically active to maintain optimal state of health.

Total amount of PA in a week positively correlated with cardiorespiratory fitness parameters, but only in women total amount of PA correlated positively with handgrip strength of right hand and $m$. rectus abdominis upper part strength and vigorous $\mathrm{PA}$ - also with oblique muscles strength in women after adjusting by gender. D. Martinez-Gomez et al. (2010) study is consistent with our finding. They found that vigorous PA and moderate-to-vigorous PA were related to CRF, but only vigorous PA was related with MF after adjusting by sex. Researchers of Netherlands and Belgium Sassen B et al. (2010) found similar results: aerobic fitness $(\mathrm{p}<0.001)$ was positively correlated with physical activity $(\mathrm{p}<0.01)$.

With regard to PA of different study programs, we have found that women of PE study program had a prevalence of high PAL, comparing with prevalence of moderate PA in women of PT study program and that men of PE study program were more physically active than men of PT study program, having a prevalence of high PAL. These findings are consistent with previous research of K. Gorner et al. (2009). The female and male students of Physiotherapy characterized a much lower level of PA in comparison to the female and male students of Physical Education (K. Gorner, 2009). This could be explained by the essence of additional sport activities of PE students during their studies at university.

Researchers from Taiwan Cheng et al. (2011) concluded that college students who routinely engage in exercise tend to be heavier, have superior muscles strength and stamina, and have better flexibility than those who do not exercise on a regular basis. As in our results high PA level was an indicator of regular sport exercises, we can conclude that we have found similar results and data analysis showed that students with high PAL had higher body weight, had better abdominal muscles strength and handgrip strength. However, flexibility of hamstring muscles didn't show statistically significant association with PAL.

Our findings are also consistent with Beets $M$. et al. (2005) research results. They noted a positive increase in cardiovascular fitness with the increase of taken sport activities. They found that adolescents enrolled in PE and participating in one or more school-sponsored sports exhibited greater cardiovascular fitness levels than adolescents participating solely in PE classes.

This study has some limitations. First, the study sample consisted of different amount of students of both genders and study programs. The study sample should be widened and almost equivalent amount of men and women of Physiotherapy and Physical Education study programs should be selected in order to make stable conclusions and also additional associations may be found. However, results of this study were analyzed taking into account this limitation. Secondly, the sample was composed of apparently healthy individuals with large group of people with normal body composition parameters; this may have influenced the results obtained for health-related physical fitness. Thus, generalization of these conclusions is only valid among apparently healthy young adults and mostly women. 
Int. Conf. SOCIETY. HEALTH. WELFARE.

\section{Conclusions}

1) Body composition values, hamstrings and $m$. quadratus lumborum elasticity were normal in most of the students.

2) Women were less physically active that men, and physical activity level was higher in Sport Education students than Physiotherapy students in both genders.

3) Greater physical activity generally implies a higher level of health-related physical fitness.

4) Higher physical activity level has positive influence on abdominal muscles strength, handgrip strength, and aerobic fitness.

\section{References}

[1] ACSM (2010). ACSM's health-related physical fitness assessment manual. - 3rd ed. / editor, Leonard Kaminsky. - Philadelphia: Lippincott: Williams \& Wilkins. 2, 4-5. pp

[2] Adams, G.M., Beam, W.C. (2008). Exercise physiology laboratory manual, 5th ed., McGraw-Hill, New York, 47-48, 153, 261-262. pp

[3] Beets, W.M., \& Piletti, K.H. (2005). Contribution of Physical Education and Sport to HealthRelated Fitness in High School Students. Journal of School Health, 75 (1), 25, 29

[4] Boreham, C., Twisk, J., Murray, L., Savage, M., Strain, J.J., Cran, G. (2001). Fitness, fatness, and coronary heart disease risk in adolescents: the Northern Ireland Young Hearts Project. Med Sci Sports Exerc. 33, 270-274

[5] Boreham, C., Twisk, J., Neville, C., Savage, M., Murray, L., Gallagher, A. (2002). Associations between physical fitness and activity patterns during adolescence and cardiovascular risk factors in young adulthood: The Northern Ireland Young Hearts Project. Int J Sports Med. 23, Suppl 1, 22-26

[6] Cheng, J.S, Yang, M.C., Ting, P.H., Chen, W.L., Huang, Y.Y. (2011). Leisure, lifestyle, and healthrelated physical fitness for college students. Social Behavior and Personality. 39 (3), 324, 330-331

[7] Ekelund, U., Poortvliet, E., Nilsson, A., Yngve, A., Holmberg, A., Sjostrom, M. (2001). Physical activity in relation to aerobic fitness and body fat in 14- to 15-year-old boys and girls. Eur J Appl Physiol. 85, 195-201

[8] Görner, K., Boraczyński, T., Štihec, J. (2009). Physical activity, body mass, body composition and the level of aerobic capacity among young, adult women and men. Sport SPA. 6 (2), 8-10

[9] Hasselstrom, H., Hansen, S.E., Froberg, K., Andersen, L.B. (2002). Physical fitness and physical activity during adolescence as predictors of cardiovascular disease risk in young adulthood. Danish youth andsports study. An eight-year follow-up study. Int J Sports Med. 23, Suppl 1, 27-31

[10] Janz, K.F., Dawson, J.D., Mahoney, L.T. (2002). Increases in physical fitness during childhood improve cardiovascular health during adolescence: the Muscatine Study. Int J Sports Med. 23, Suppl 1, 15-21

[11] Kundziòa, I., Grants, J. Sedentary living and the level of physical activity in Latvia: analysis and problem. Journal of Sport Science. Nr. 2011, 2 (1), 85

[12] Lynn, P. M., \& Epler, M. E. (1998). Fundamentals of musculosceletal assessment techniques, 2nd ed. New-York: Lippincott. 243-244, 290-291

[13] Martinez-Gomez, D., Eisenmann, J.C., Gomez-Martinez, S., Veses, A., Romeo, J., Veiga, O.L., Marcos, A., AFINOS Study Group. (2010). Associations of physical activity and fitness with adipocytokines in adolescents: The AFINOS study. Nutrition, Metabolism \& Cardiovascular Diseases. 22 (3): 255. DOI:10.1016/j.numecd.2010.07.010

[14] Mesa, J.L., Ortega, F.B., Ruiz, J.R., Castillo, M.J., Hurtig-Wennlöf, A., Sjöström, M., Gutiérrez, Á. (2006). The importance of cardiorespiratory fitness for healthy metabolic traits in children and adolescents: the AVENA Study. J Public Health. 14, 178-179. DOI 10.1007/s10389-006-0033-9 


\section{SHS Web of Conferences}

[15] Moliner-Urdiales, D., Ruiz, J.R., Ortega, F.B., Jimenez-Pavon, D. et al. on behalf of the AVENA and HELENA Study Groups. Secular trends in health-related physical fitness in Spanish adolescents: The AVENA and HELENA Studies. Journal of Science and Medicine in Sport, 13: 584. DOI: $10.1016 /$ j.ksams.2010.03.004

[16] Nielsen, G.A., Andersen, L.B. (2003). The association between high blood pressure, physical fitness, and body mass index in adolescents. Prev Med. 36, 229-234

[17] Ortega, F.B., Ruiz, J.R., Castillo, M.J., Moreno, L.A., Gonzalez-Gross, M., Wärnberg, J., Gutiérrez, I. and the AVENA Group. (2005). Low Level of Physical Fitness in Spanish Adolescents. Relevancefor Future Cardiovascular Health (AVENA Study). Rev Esp Cardiol. 58 (8), 899

[18] Patterson, E. (2010, November). Guidelines for Data Processing and Analysis of the International Physical Activity Questionnaire (IPAQ). Retrieved April 12, 2013, from https://sites.google. com/site/theipaq/scoring-protocol

[19] Porozovs, J., Porozova, Dz., Valdemiers, A. (2012). Jauniešu fiziskās aktivitātes un veselības problēmas [The Physical Activities and Health Problems of Young People] Scientific Journal of Riga Technical University. 20, 95

[20] Sassen, B., Kok, G., Schaalma, H., Kiers, H., Vanhees, L. Cardiovascular risk profile: Crosssectional analysis of motivational determinants, physical firness and physical activity. BioMed Central Public Health, 10, 592

[21] Twisk, J.W., Kemper, H.C., Van Mechelen, W. (2002). Prediction of cardiovascular disease risk factors later in life by physical activity and physical fitness in youth: general comments and conclusions. Int J Sports Med. 23, Suppl 1, 44-49

[22] Wedderkopp, N., Froberg, K., Hansen, H.S., Riddoch, C., Andersen, L.B. (2003). Cardiovascular risk factors cluster in children and adolescents with low physical fitness: The European Youth Heart Study (EYHS). Pediatr Exerc Sci. 15, 419-27 